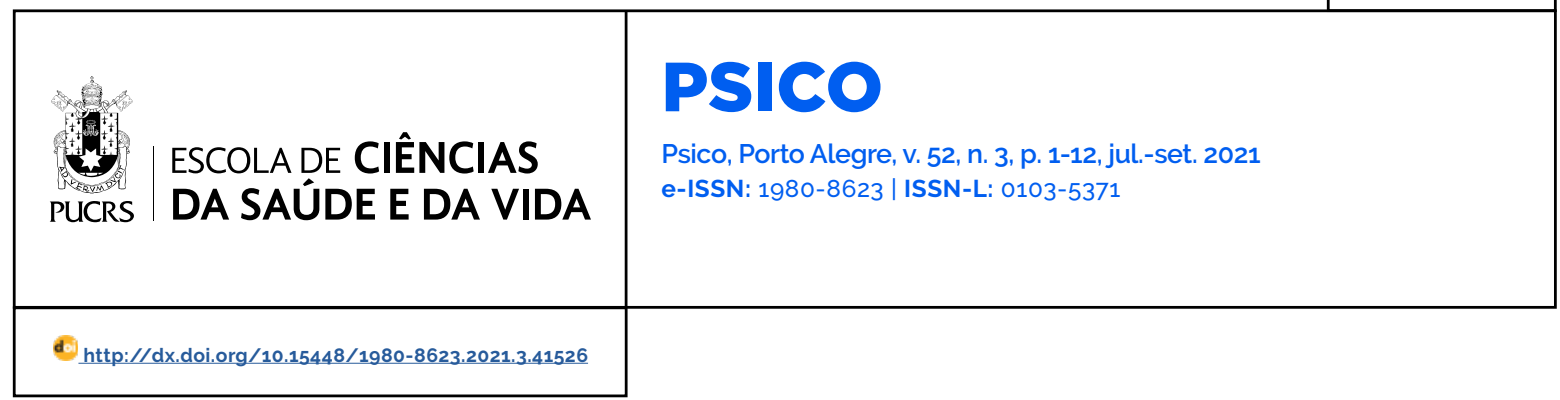

SEÇÃO: ARTIGO

\title{
Uma intervenção de Terapia Focada na Compaixão em grupos online no contexto da pandemia por COVID-19 ${ }^{1}$
}

\author{
Compassion Focused Therapy Online in Groups in the context of the COVID-19 \\ pandemic \\ Una intervención Terapia Basada en la Compasión en Grupos Online en el contexto de \\ la pandemia por COVID-19
}

\section{Nazaré de Oliveira \\ Almeida $^{2}$ \\ orcid.org/0000-0001-8610-3753 \\ nazareacademico@gmail.com}

\section{Isabela Pizzarro \\ Rebessi ${ }^{2}$ \\ orcid.org/0000-0002-5921-8536 \\ isabela.rebessi@usp.br}

\section{Karen Priscila Del Rio}

\section{Szupszynski ${ }^{3}$}

orcid.org/0000-0001-7726-8097

karenszu@yahoo.com.br

\section{Carmem Beatriz \\ Neufeld ${ }^{2}$}

orcid.org/0000-0003-1097-2973 cbneufeld@usp.br

Recebido em: 13 ago. 2021 Aprovado em: 24 ago. 2021. Publicado em: 27 out. 2021.

\section{c) (i)}

Artigo está licenciado sob forma de uma licença Creative Commons Atribuição 4.0 Internacional.
Resumo: A Terapia Focada na Compaixão (TFC) é uma abordagem de tratamento transdiagnóstico que visa desenvolver capacidades de calma e de afiliação como uma maneira de regular o sistema de ameaças, o que pode ser muito útil em situação de crise, como o caso da pandemia pelo COVID-19. Trata-se de um relato de experiência que tem como objetivo apresentar uma intervenção em grupo de três sessões semanais de TFC realizada em ambiente virtual, com objetivo de oferecer suporte à população na pandemia por COVID-19, avaliando possiveis mudanças nos niveis de depressão, ansiedade, estresse e autocompaixão. Neste estudo, 106 participantes concluiram a intervenção ofertada dentro do programa "LaPICC contra COVID-19". Foram encontradas diferenças significativas nas medidas quantitativas de depressão, ansiedade e estresse, além da autocompaixão. Os dados qualitativos também indicaram mudanças em termos de compaixão e de autocompaixão. Esses achados sugerem que a compaixão pode contribuir para a saúde mental e bem-estar psicológico, bem como para o enfrentamento de crises nos participantes deste grupo. Palavras-chave: terapia focada na compaixão, compaixão, covid-19, lapicc contra covid, intervenção online em grupo

Abstract: Compassion Focused Therapy (CFT) is a transdiagnostic treatment approach that aims to develop calm and affiliative capacities as a way to regulate the threat system, which can be very useful in crisis situations, as in the case of the COVID-19 pandemic. This is an experience report that aims to present a group intervention of 3 weekly sessions of CFT carried out online, in order to support the population in the COVID-19 pandemic, evaluating possible changes in the levels of depression, anxiety, stress and self-compassion. In this study, 106 participants completed the intervention offered within the "LaPICC against COVID-19" program. Significant differences were found in the quantitative measures of depression, anxiety and stress, in addition to self-compassion. Qualitative data also indicated changes in terms of compassion and self-compassion. These findings suggest that compassion can contribute to mental health and psychological well-being, as well as to coping with crises in the participants of this group.

Keywords: compassion focused therapy, compassion, covid-19, lapicc against covid, online group interventions

Resúmen: La Terapia Basada en la Compasión (CFT) es un abordaje de tratamiento transdiagnóstico que tiene como objetivo desarrollar capacidades de calma y afiliación como una forma de regular el sistema de amenazas, lo que puede ser de gran utilidad en situaciones de crisis, como es el caso de la pandemia del COVID -19. Se trata de un relato de experiencia que tiene como objetivo presentar una intervención grupal de 3 sesiones semanales de TFC, realizadas en línea, con el fin de apoyar a la población en la pandemia del CO-

\footnotetext{
Apoio financeiro: CNPq e CAPES

Universidade de São Paulo (USP-RP), Ribeirão Preto, SP, Brasil.

Pontifícia Universidade Católica do Rio Grande do Sul (PUCRS), Porto Alegre, RS, Brasil.
} 
VID-19, evaluando posibles cambios en los niveles de depresión, ansiedad, estrés y autocompasión. En este estudio, 106 participantes completaron la intervención ofrecida dentro del programa "LaPICC contra COVID-19". Se encontraron diferencias significativas en las medidas cuantitativas de depresión, ansiedad y estrés, además de la autocompasión. Los datos cualitativos también indicaron cambios en términos de compasión y autocompasión. Estos hallazgos sugieren que la compasión puede contribuir a la salud mental y el bienestar psicológico, así como hacer frente a las crisis en los participantes de este grupo.

Palabras clave: terapia basada en la compasión, compasión, terapia online, covid-19, lapicc contra covid-19

Em dezembro de 2019 um novo coronavírus de alta transmissibilidade, nomeado "SARSCoV-2", e cuja infecção foi denominada "COVID-19" (Corona Virus Disease), rapidamente levou agências globais de saúde pública, como a Organização Mundial da Saúde (OMS) e o Centro de Controle e Prevenção de Doenças (CDC) a reconhecerem seu potencial pandêmico (Lu et al., 2020, Du Toit, 2020; Huang et al., 2020; Zu et al., 2020). Depois do anúncio da China acerca do estado da epidemia no país, bem como da sua prevenção, a OMS anunciou o distanciamento social como uma das medidas eficazes e diretas para impedir a transmissão da doença. A partir disso, uma crise global de saúde pública se instalou no mundo, com número crescente de casos, cujo impacto socioeconômico, político e psicossocial levou muitos países a condições de caos em seus sistemas de saúde (Du Toit, 2020). Isso porque, devido à alta transmissibilidade do vírus e aos sintomas respiratórios que, muitas vezes, demandam tratamento intensivo, não havia equipamentos disponíveis para serem adquiridos (mesmo que esses sistemas tivessem recursos financeiros para o aumento de sua estrutura hospitalar). Desta forma, a medida para conter a propagação da infecção foi a quarentena obrigatória de toda a população, não apenas de casos afetados, ou seja, bilhões de pessoas (Lu et al., 2020). Na mesma direção, quando as vacinas foram testadas e aprovadas, a confecção, a distribuição e a disponibilidade de matéria prima também afetaram o curso das medidas de prevenção.

No que se refere aos efeitos psicológicos do distanciamento social, a solidão e o tédio, agravados pela ansiedade e pelo pânico em massa gerados pelo desconhecimento da duração da pandemia, podem ser considerados efeitos mais frequentes. Benerjee e Rai (2020) afirmam que uma crise como essa que vivemos geralmente afeta a mente humana de maneira crucial, aumentando a sensação de ameaça e provocando uma "bola de neve" na ansiedade. Os autores chamam atenção para a negligência dos efeitos psicológicos decorrentes de uma pandemia.

Diante do contexto pandêmico e da necessidade do distanciamento social, um fenômeno em relação ao uso de tecnologia surgiu como alternativa, mas que era visto com resistência pela maioria das pessoas de diferentes contextos profissionais e sociais, a despeito da opinião dos especialistas na área. A digitalização mostrou a possibilidade da realização de muitas das atividades antes vistas com desconfiança na modalidade online, entre elas as intervenções psicológicas (Conselho Federal de Psicologia [CFP], 2012, 2020).

Sendo assim, as restrições impostas pela pandemia da COVID-19 também afetaram o trabalho dos profissionais de psicologia, levando-os à necessária adaptação de seu trabalho ao ambiente virtual. No entanto, é importante ressaltar que o uso da tecnologia em psicologia já era praticado e pesquisado muito antes da pandemia da COVID-19. As intervenções online em saúde são conhecidas como e-Health e podem ser definidas como serviços ou informações em saúde oferecidos por meio da internet ou tecnologias relacionadas (Bedendo, 2019). A e-Health tem sido amplamente utilizada por demonstrar eficácia para um grande número de problemas, como: transtornos de ansiedade, obesidade, transtornos alimentarese dor crônica e problemas de fadiga (Andersson \& Carlbring, 2017). São inúmeras as vantagens de serviços disponibilizados de forma online, entre elas: facilidade de acesso e de implementação de serviços, baixo custo, disponibilidade de acesso 24 horas por dia, maior adesão pela baixa exposição do paciente, entre outras (Voogt et al., 2012).

Não obstante, também existem os dificultadores do trabalho realizado de forma virtual. Um exemplo disso é a restrição da comunicação não verbal, presente no corpo do cliente e do terapeuta, bem como a dificuldade de trabalhos de movimentação 
dos corpos e das cadeiras, usadas principalmente em terapias vivenciais. Este é o caso da Terapia Focada na Compaixão (TFC) (Neff, 2020).

A TFC foi desenvolvida por Gilbert (2009) como uma abordagem de tratamento transdiagnóstico que visa criar autocompaixão e reduzir o sentimento de vergonha, desenvolvendo um sistema de suporte interno que precede o envolvimento com o conteúdo interno doloroso (Gilbert \& Choden, 2014). A psicoeducação na perspectiva neurocientífica e evolutiva da mente é um elemento essencial para a TFC. Gilbert (2009) baseou-se nos conceitos evolutivos de sistemas de autorregulação de Depue e Morrone-Strupinsky (2005), segundo os quais diferentes motivações são reguladas por emoções que, por sua vez, são reguladas por três sistemas: o sistema de detecção, defesa e proteção (sistema de ameaças), o sistema de recursos, aquisição e conquista (sistema Drive) e o sistema de segurança, cuidado e satisfação (sistema calmante).

A TFC estimula as capacidades de calma e de afiliação como uma maneira de regular o sistema de ameaças. O modelo evolucionista que a sustenta considera o sentimento de vergonha e de autocriticismo essenciais para a manutenção da psicopatologia (Gilbert \& Procter, 2006; Gilbert, 2014, 2015). Em situações de ameaça social, abuso ou hostilidade, o autocriticismo é uma estratégia adaptativa associada ao sentimento de vergonha, derrota, rejeição e perseguição social, tendo sua origem em memórias de eventos ameaçadores. Dois problemas decorrem do autocriticismo: o conteúdo dos pensamentos e o tipo/intensidade das emoções direcionadas ao self, somados ao fracasso do sistema calmante. Diante dessa situação, Gilbert guiou o foco da pesquisa sobre TFC à relação entre os pensamentos alternativos dos pacientes e sentimentos de decepção, hostilidade, desprezo ou abandono, seguidos por suas intervenções experimentais com tons emocionais favoráveis e úteis para novos pensamentos alternativos (Gilbert \& Procter, 2006; Gilbert, 2014, 2015). Como resultado, Gilbert (2009) observou a associação de estados de vergonha e de autocrítica com desconforto e resistência, incorporando técnicas de cultivo de uma atitude de autobondade para indivíduos com altos níveis de vergonha e de autocrítica. Em geral, estudos sobre a eficácia da TFC mostraram que, após a intervenção, os pacientes mostram uma redução significativa nos sintomas depressivos, de ansiedade, de vergonha, de autocrítica e de comportamentos submissos, além de um aumento na capacidade de autocontrole e de compaixão pelo eu (Gilbert \& Procter, 2006; Gilbert, 2014, 2015).

Até o momento, pouco se tem pesquisado sobre intervenções baseadas na TFC realizadas em ambiente online. Um dos exemplos é o estudo de Krieger et al. (2018) realizado na Suiça e no qual 122 participantes com nivel significativo de autocrítica foram submetidos a uma intervenção focada na compaixão, com o objetivo de avaliar sua eficácia. Outro estudo foi o de Kelly et al. (2010), que investigou uma população de 119 fumantes, por meio de uma intervenção experimental com exercicios de imagem desenhadas de fala interna para estimular o sistema clamante, com dois grupos, um grupo experimental (imagens autoenergizantes e autocontroladas) e um grupo controle (automonitoramento diário). Outro exemplo é a pesquisa de Shapira e Mongrain (2010), que recrutou uma amostra não clínica de 1.002 participantes usando a internet, administrando uma intervenção experimental de escritas de cartas compassivas e visualização de cenas de otimismo, comparada a uma condição controle, na qual os participantes escreveram livremente sobre uma memória inicial. Todos esses pesquisadores encontraram sintomas diminuídos de depressão, ansiedade e estresse, bem como melhora nos estados de autocompaixão, autocritica, mindfulness, satisfação com a vida, medo da autocompaixão, autoestima e vergonha existencial a partir de intervenções online.

O desenvolvimento da autocompaixão proposto pela TFC desativa o sistema comumente ativado diante de situações estressantes, como o distanciamento social e ativa o sistema de calma que regula as emoções (Gilbert \& Irons, 2005). Por ser focado na afiliação, esse sistema estimula a busca de conexão interna e com os outros, mesmo que as formas sejam virtuais, o que pode 
facilitar as intervenções de TFC na forma online. Logo, a intervenção em TFC no contexto pandêmico pode ser de extrema ajuda na regulação das emoções desconfortáveis vivenciadas nesse período, trazendo possiveis benefícios em relação aos efeitos psicológicos do distanciamento social provocado pela pandemia. A partir disso, o objetivo desse trabalho é trazer um relato de experiência de uma intervenção de três semanas de TFC em grupos (TFCG) realizada em ambiente virtual para participantes que apresentavam sintomas de ansiedade, depressão e estresse relacionados com a pandemia de COVID-19. O presente trabalho trata-se de uma intervenção ofertada pelo programa LaPICC contra COVID-19 como uma forma de mitigar os sofrimentos causados pela pandemia em nosso País.

\section{Método}

\section{Participantes}

Para a fase de intervenção, 142 participantes foram selecionados por conveniência, de acordo com a ordem de chegada, disponibilidade e aceitação de participação, sendo a eles garantido o direito de desistência da participação a qualquer momento durante a intervenção. A amostra final do presente relato de experiência foi composta por 106 participantes. Dados detalhados das características sociodemográficas podem ser encontrados na Tabela 1.

Tabela 1 - Caracterização sociodemográfica dos participantes da amostra total

\begin{tabular}{lccc}
\hline & Total & Completaram & Desistiram \\
\hline N & 142 & $106(74 \%)$ & $36(26 \%)$ \\
$\quad$ Idade média (anos) & 39,91 & 41,20 & 36,06 \\
Desvio Padrão (DP) & $(10,32)$ & $(9,78)$ & $(10,32)$ \\
Sexo & & & $25(20,7 \%)$ \\
$\quad$ Feminino & 121 & $96(79,3 \%)$ & $11(52,4 \%)$ \\
Masculino & 21 & $10(47,6 \%)$ & $21(51,2 \%)$ \\
Escolaridade & & $20(48,8 \%)$ & $15(14,9 \%)$ \\
$\quad$ Ensino Superior incompleto & 41 & $86(85,1 \%)$ & $26(60,5 \%)$ \\
Ensino Superior completo & 101 & $17(39,5 \%)$ & $4(26,7 \%)$ \\
Uso de medicamento psiquiátrico & 43 & $11(73,3 \%)$ & $3(8,4 \%)$ \\
Acompanhamento psiquiátrico & 15 & $33(91,6 \%)$ & \\
Realiza acompanhamento psicológico & 36 & & \\
Profissão dos participantes & & & \\
Profissionais de saúde & $99(93,3 \%)$ & & \\
Profissionais de outras áreas & $07(6,7 \%)$ & & \\
\hline
\end{tabular}

Os critérios de inclusão foram: ser adulto, ter ensino fundamental completo, e apresentar sintomas de depressão, ansiedade ou estresse. A avaliação dos desses foi realizada por meio da aplicação da DASS-21, sendo que os participantes que pontuavam extremamente grave para ansiedade, depressão e estresse não foram incluídos no grupo. Além disso, também eram excluidos participantes que relataram transtornos mentais em estado agudo, avaliados por um questionário sociodemográfico. Os índices de autocompaixão não foram utilizados como critério de inclusão ou de exclusão dos participantes no grupo. Os participantes com diagnósticos de transtornos mentais em estado agudo foram encaminhados para uma rede de atendimento individual online. Vale ressaltar que, apesar de não ser critério de inclusão, a profissão dos participantes foi, em sua maioria, de profissionais de saúde. 


\section{Instrumentos}

Para avaliação dos participantes foram utilizados os seguintes instrumentos:

1. Questionário sociodemográfico. Foi elaborado um questionário a fim de reunir dados de caracterização da amostra. As perguntas do questionário diziam respeito sobre o nome, contatos do participante (e-mail e número de celular), sexo, idade, escolaridade, profissão, convênio de saúde, quais serviços eram procurados quando um atendimento de saúde era necessário, se a pessoa estava em algum tipo de tratamento ou possuía algum dos seguintes diagnósticos: câncer, dor crônica, epilepsia, Transtorno Depressivo, Transtorno de Ansiedade, Transtorno Bipolar, Transtorno Esquizofrênico. Também foi questionado se os participantes faziam acompanhamento médico ou psicológico.

2. Depressiom, Anxiety and Stress Scale (DASS-21). Foi utilizada a DASS-21 para avaliar sinais de depressão, ansiedade e estresse. Ela foi desenvolvida por Lovibond e Lovibond (1995) e é um conjunto de três subescalas do tipo Likert de 4 pontos, com pontuação de o a 3. A pontuação máxima da escala total é de 63 e, a pontuação máxima por subitem é 21. Foi adaptada para o contexto brasileiro por Vignola e Tucci (2014), que encontraram uma boa consistência interna: alfa de Cronbach de 0,95 para o total das três subescalas.

\section{Self-Compassion Scale (SELFCS ou SCS).}

A escala foi desenvolvida por Neff (2003) adaptada para o contexto brasileiro por Souza e Hutz (2016) e é composta por 26 itens que devem ser respondidos, a partir de uma escala Likert de 5 pontos, quanto à atitude do individuo consigo mesmo em momentos dificeis e de sofrimento. Esses itens dividem-se entre seis subescalas: Bondade consigo; Autocrítica severa; Senso de humanidade; Isolamento; Mindfulness; e Sobreidentificação. O escore total é adquirido a partir da soma de todas as subescalas, sendo que os escores das subescalas de autojulgamento, isolamento e sobreidentificação são invertidos. O escore máximo e mínimo por subescala é: Bondade consigo 5 e 25: Autocrítica severa 5 e 25: Senso de humanidade 4 e 20; Isolamento 4 e 20; Mindfulness e Sobreidentificação 4 e 20. 0 escore máximo e mínimo por subescala é 26 e 130. Dessa forma, quanto maior o escore total, mais autocompassivo o indivíduo.

4. Régua da ansiedade. Foi utilizada uma Escala Visual Analógica, a qual era aplicada ao final de cada sessão. Os participantes foram instruidos a responder a seguinte pergunta: "Em uma escala de o a 10, sendo o nada ansioso e 10 extremamente ansioso, como você se sente agora?".

5. Questões qualitativas. Devido ao fato de não haver nenhuma escala que avalie os efeitos da TFC validada para o contexto brasileiro, foram elaboradas seis questões qualitativas, as quais foram inspiradas na Escala de Engajamento e Ação Compassivas (Gilbert et al., 2017). As questões foram apresentadas na última sessão, sendo elas:

1. Comente com qual frequência você percebe e é sensivel ao seu sofrimento.

2. Comente com qual frequência você cria sentimentos internos de apoio, utilidade e encorajamento.

3. Comente com qual frequência você percebe e é sensivel ao sofrimento dos outros quando ela surge.

4. Comente com qual frequência você expressa sentimentos de apoio, ajuda e encorajamento para as outras pessoas.

5. Comente com qual frequência os outros percebem e são sensiveis ao seu sofrimento quando surge em você.

6. Comente com qual frequência os outros te tratam com sentimentos de apoio, utilidade e encorajamento.

\section{Procedimentos}

Os grupos foram formados a partir de um cadastro realizado de forma online e por meio de um formulário (Google Forms) no qual os participantes assinavam o TCLE, e preenchiam o questionário sociodemográfico e a DASS-21. A divulgação dos grupos foi realizada via redes sociais digitais do LaPICC (Laboratório de Pesquisa e Intervenção Cognitivo Comportamental) do Departamento de Psicologia da Faculdade de Filosofia, Ciências e 
Letras de Ribeirão Preto da Universidade de São Paulo. Neste projeto foram ofertadas diferentes intervenções para sintomas de ansiedade, depressão e estresse relacionados com a pandemia do COVID-19 (para conhecer o projeto como um todo e os outros grupos ofertados consultar Neufeld et al (2021). Neste estudo, foram avaliados os efeitos da intervenção de três sessões semanais de TFC.

Após preencherem o formulário online, contendo o questionário sociodemográfico e a DASS-21 (Google Forms), os dados eram redirecionados para uma planilha conforme a ordem de preenchimento. Após a análise dos critérios de inclusão e de exclusão, os participantes selecionados para a participação no grupo de TFC eram contatados pela terapeuta do grupo, via telefone ou e-mail, para confirmar o interesse e, também, verificar a disponibilidade de participação nos horários que eram ofertados. Caso os participantes não tivessem disponibilidade de horário, eles eram encaminhados para outro grupo ou outro atendimento.

Foram realizados 12 grupos, com média de nove participantes em cada grupo. A intervenção foi realizada ao longo do ano de 2020. Para a composição dos grupos foi utilizado o critério de conveniência, no qual os participantes deveriam ter disponibilidade de participação nos dias e horários dos grupos, e preencher os critérios de inclusão da amostra.

Uma vez que um grupo era formado, era aplicada a escala SELFCS antes da primeira sessão, durante a intervenção, e após o término. A DASS21 havia sido preenchida no começo e foi reaplicada após o término da intervenção. Além disso, os participantes foram instruídos a responder à "régua da ansiedade" após cada uma das sessões.

Todos os grupos foram conduzidos pela mesma terapeuta, que tem extensa formação e experiência em TFC e que recebeu supervisões semanais para manejo e condução dos grupos. Vale salientar que esse estudo cumpre as exigências éticas de pesquisa conforme a resolução n. 466/2012 do Conselho Nacional de Saúde e foi aprovada pelo Comitê de Ética em Pesquisa sob o protocolo n. CAAE 36482320.6.0000.5336.

O programa de três semanas consistiu em uma adaptação do protocolo de 12 semanas desenvolvido por Kirby et al. (2018) A Tabela 2 apresenta o tema central, o conteúdo e as práticas das três sessões semanais, com 2 horas de duração por sessão.

Tabela 2 - Programa de intervenção

\begin{tabular}{|c|c|c|c|}
\hline Sessão & Temas centrais & Conteúdos & Práticas e exercícios \\
\hline 1 & $\begin{array}{l}\text { - As bases evolucio- } \\
\text { nistas e neurofisioló- } \\
\text { gicas da compaixão }\end{array}$ & $\begin{array}{l}\text { - Boas-vindas } \\
\text { - Apresentação dos participan- } \\
\text { tes, incluindo a motivação de } \\
\text { estar no grupo } \\
\text { - Contrato de convivência } \\
\text { - O que é e o que não é compaixão } \\
\text { - O cérebro complicado } \\
\text { - Os três sistemas de regulação } \\
\text { do afeto ou Três tipos de emoção }\end{array}$ & $\begin{array}{l}\text { - Prática da "Posição" Compassiva } \\
\text { - Prática "Usando expressões faciais e } \\
\text { tons de voz" } \\
\text { - Prática "Ritmo de Respiração Tranqui- } \\
\text { lizante (Respiração Calmante)" }\end{array}$ \\
\hline 2 & $\begin{array}{l}\text { - Segurança } \\
\text { - Múltiplos eus }\end{array}$ & $\begin{array}{l}\text { - Busca de segurança e proteção } \\
\text { versus a segurança (sensação de } \\
\text { segurança) } \\
\text { - Múltiplos eus }\end{array}$ & $\begin{array}{l}\text { - Exercício "Explorando os Múltiplos } \\
\text { Eus em detalhes" } \\
\text { - Prática "Lugar seguro" } \\
\text { - Prática "Outro Compassivo ideal" }\end{array}$ \\
\hline 3 & $\begin{array}{l}\text { - Vergonha } \\
\text { - Autocriticismo }\end{array}$ & $\begin{array}{l}\text { - Eu Compassivo } \\
\text { - Eu crítico } \\
\text { - Vergonha } \\
\text { - Autocriticismo } \\
\text { - Assertividade compassiva } \\
\text { - Perdão }\end{array}$ & $\begin{array}{l}\text { - Exercício "Análise funcional do Eu } \\
\text { Crítico" } \\
\text { - Exercício "Explorando o Eu Compassivo" } \\
\text { - Exercício "Explorando a vergonha" } \\
\text { - Prática "Abraçando a parte crítica e a } \\
\text { parte criticada com o Eu Compassivo" }\end{array}$ \\
\hline
\end{tabular}




\section{Resultados e discussão}

Inicialmente a equivalência dos 12 grupos no pré-teste foi analisada por meio de uma ANOVA de fator único e como não foram encontradas diferenças significativas (ps>0,2) em nenhuma das variáveis no pré-teste entre os grupos, as análises subsequentes foram realizadas com o grupo total, ou seja, os 106 participantes que finalizaram a intervenção.
Os dados de pré e pós-teste foram analisados por meio de um teste ANOVA de fator duplo com repetição e o tamanho de efeito foi calculado pelo $n^{2}$ (eta squared). As análises estatisticas foram feitas por meio do software SPSS versão 23.0.

$\mathrm{Na}$ Tabela 3 podem ser observados os resultados quantitativos obtidos através dos instrumentos anteriormente descritos.

Tabela 3 - Dados gerados a partir das medidas de resultado pré e pós-intervenção $(n=106)$

\begin{tabular}{|c|c|c|c|c|c|c|c|}
\hline & \multicolumn{2}{|l|}{ PRE } & \multicolumn{2}{|l|}{ POS } & \multicolumn{2}{|c|}{$\begin{array}{l}\text { Anova fator duplo com } \\
\text { repetição }\end{array}$} & \multirow[b]{2}{*}{ Interpretação } \\
\hline & M & DP & M & DP & $p$ & $\begin{array}{c}n^{2}(e t a \\
\text { squared })\end{array}$ & \\
\hline Depressão (DASS) & 15,74 & 2,40 & 10,47 & 1,65 & $8,86 \mathrm{E}-37^{* *}$ & 0,431 & Médio \\
\hline Ansiedade (DASS) & 12,37 & 1,25 & 6,88 & 0,79 & $4,81 \mathrm{E}-61^{* *}$ & 0,076 & Pequeno \\
\hline Estresse (DASS) & 20,58 & 2,13 & 10,82 & 3,16 & $4.39 \mathrm{E}-38^{* *}$ & 0,575 & Médio \\
\hline B. Consigo & 4,27 & 2,02 & 18,56 & 1,24 & $7,8 \mathrm{E}-105^{\star *}$ & 0,399 & Médio \\
\hline Autoc. Sever & 12,88 & 4,66 & 3,82 & 3,10 & $1,58 \mathrm{E}-37^{\star *}$ & 0,694 & Grande \\
\hline Senso Hum. & 4,03 & 2,15 & 11,27 & 4,15 & $5.92 \mathrm{E}-37^{* *}$ & 0,546 & Médio \\
\hline Isolamento & 11,18 & 4,06 & 3.50 & 1,42 & $8,16 \mathrm{E}-46^{* *}$ & 0,343 & Médio \\
\hline Mindfulness & 6,31 & 2,64 & 16,52 & 2,62 & $5,17 \mathrm{E}-73^{* *}$ & 0,474 & Médio \\
\hline Sobre-ident. & 16,73 & 1,75 & 4,44 & 1,24 & $7,6 \mathrm{E}-124^{* *}$ & 0,149 & Pequeno \\
\hline Régua 1 & 7,61 & 2,02 & 3,61 & 2,02 & $8,87 \mathrm{E}-37^{* *}$ & 0,001 & Pequeno \\
\hline Régua 2 & 7,56 & 1,51 & 2,56 & 1,51 & $2,37 \mathrm{E}-44^{* *}$ & 0,001 & Pequeno \\
\hline Régua 3 & 7,40 & 1,98 & 2,40 & 1,98 & $1,35 E-53^{* *}$ & 0,001 & Pequeno \\
\hline
\end{tabular}

Legenda: **diferenças significativas com valores de $p<0,0001$

Interpretação: Ferguson (2009)

De acordo com os dados, a média de respostas dos participantes à escala DASS-21 demonstra que eles apresentavam um nivel moderado de depressão, ansiedade e estresse. Depois das três sessões de TFC esses niveis melhoraram significativamente, com tamanhos de efeito médio para depressão e estresse, e pequeno para ansiedade. A resposta à "régua da ansiedade" confirmou a redução da ansiedade percebida, o que ocorreu ao longo das sessões. A TFC estimula as capacidades de calma e de afiliação e com isso regula o sistema de ameaça. Isso pode explicar o fato de os participantes terem melhorado significativamente os niveis de depressão, ansiedade e estresse. Ter uma sensação positiva de bem-estar, satisfação e segurança estão fortemente associadas a niveis menores de depressão, ansiedade e estresse (Gilbert et al., 2008). No entanto, cabe ressaltar que por se tratar de um relato de experiência, apenas se pode afirmar que foram encontradas mudanças, e levantar a hipótese de que elas tenham relação com a intervenção proposta, sem, no entanto, poder afirmar com certeza que as mudanças observadas ocorreram exclusivamente devido à intervenção ofertada. 
Sugere-se que seja de grande relevância que a mudança tenha ocorrido com apenas três sessões, exceto se considerarmos o fato que para alguns autores (Demarzo et al., 2017) o número de sessões não influencia tanto quanto o engajamento nas atividades propostas dentro e fora da sessão. Cabe ressaltar que não foram feitas medidas de engajamento, porém foi percebido comprometimento nas atividades realizadas durante as sessões e a melhora nos resultados de acordo com o autorrelato dos participantes.

A avaliação da autocompaixão por meio da Escala de Autocompaixão (Souza \& Hutz, 2016) mostrou na avaliação inicial um nivel baixo de autocompaixão, o qual também melhorou significativamente depois das três sessões de TFC, com tamanhos de efeito pequeno para sobreidentificação, médio para bondade consigo, sendo de humanidade, isolamento e mindfulness, e grande para autocrítica severa. As seis subescalas (Bondade consigo, Autocrítica severa, Senso de humanidade, Isolamento, Mindfulness e Sobreidentificação) demonstram nivel inicial baixo de autocompaixão e aumento nas subescalas, especialmente na de Bondade Consigo, que ficou 4,5 vezes maior. As outras subescalas também tiveram expressivo aumento de autocompaixão. Como citado, a eficácia da TFC está associada à redução significativa dos sintomas de depressão, ansiedade, vergonha, autocriticismo, além da capacidade de autoregulação e autocompaixão (Gilbert \& Procter, 2006; Gilbert, 2014, 2015). Apesar destes resultados, mais uma vez, cabe ressaltar que o método empregado não permite que se afirme que as mudanças ocorridas se devem à intervenção.

Para a avaliação qualitativa das questões inspiradas na Escala de Engajamento e Ação Compassivas (Gilbert et al., 2017) foi utilizada a metodologia da análise de conteúdo (Bardin, 1977). Buscou-se termos frequentes e similares, considerando-os padrões das respostas, os quais foram transformados em categorias e, posteriormente, quantificados. Essa avaliação também confirmou os baixos niveis iniciais de autocompaixão observados na Escala de Autocompaixão.
Em relação à questão 1 "Comente com qual frequência você percebe e é sensivel ao seu sofrimento", 87\% (92) das respostas contemplaram raramente e que consideravam até duvidoso ser sensivel ao próprio sofrimento. Contudo, no pós-teste as falas dos participantes parecem indicar que eles notaram o quanto precisam ser mais sensiveis ao seu sofrimento e que o contrário se refere ao medo da compaixão. Como afirma a literatura (Gilbert, 2013) é comum ouvir dos participantes durante o treinamento da mente compassiva que eles nunca pensaram que deveriam prestar atenção e dedicarem-se a si mesmos, afirmando ser isso uma total novidade. A partir disso, hipotetiza-se que, com a compreensão e a experiência da compaixão, eles sentem essa necessidade de reavaliarem determinadas percepções.

Em relação à questão 2 "Comente com qual frequência você cria sentimentos internos de apoio, utilidade e encorajamento", 63\% (67) dos participantes afirmaram que às vezes oferecem algum apoio para si mesmos, sendo que o restante afirmou que raramente o faziam. Contudo, no pós-teste os participantes relataram a percepção de que precisam se dedicar a si mesmos e que isso pode estar altamente relacionado com o bem-estar e qualidade de vida. Para Gilbert (Gilbert et al., 2012; Gilbert, 2013, 2019) esse é um dos maiores desafios da TFC, pois autocuidado, respeito aos próprios limites e criar um ambiente interno de apoio são, muitas vezes, consideradas manifestações de egoísmo ou simplesmente um ato dispensável. É comum percebermos conflito quando os participantes se confrontam com a necessidade de priorizar o autocuidado, em detrimento do cuidado aos outros (Gilbert, 2019). Os resultados apresentados nas avaliações quantitativa e qualitativa corresponderam às hipóteses traçadas sobre a presença desse "conflito" na sessão 3 acerca.

Em relação à questão 3 "Comente com qual frequência você percebe e é sensivel ao sofrimento dos outros quando ele surge", 91\% (96) dos participantes consideraram que são frequentemente sensiveis ao sofrimento dos outros. Em relação à 4 "Comente com qual frequência você expressa 
sentimentos de apoio, ajuda e encorajamento para as outras pessoas", os participantes, em sua maioria (89\%), responderam que frequentemente expressam apoio. Contudo, no pós-teste os participantes relataram a percepção de que podem manter a sua sensibilidade e engajamento no alivio do sofrimento dos outros, mas sem que isso se torne um sacrificio das próprias necessidades. Além disso, também relataram perceber em si uma dificuldade de colocar limites nas demandas dos outros em relação a eles, o que na percepção deles o programa os incentivou a mudar. Gilbert (2019) e Neff (2003) comentam o quão no ocidente é mais comum a compaixão pelos outros e incomum a compaixão para si mesmo.

Em relação à questão 5 "Comente com qual frequência os outros percebem e são sensiveis ao seu sofrimento quando surge em você", 34\% (36) responderam que raramente os outros se mostravam sensiveis, mas em torno de $71 \%$ (25) desses perceberam que podem estar com expectativas altas em relação aos outros. Entre os outros $66 \%$ (70), 31\% (22) não respondeu e 35\% (24) consideram que recebe mediano apoio dos outros. E em relação à questão 6 "Comente com qual frequência os outros te tratam com sentimentos de apoio, utilidade e encorajamento", 33\% (35) dos participantes relataram receber medianamente apoio dos outros e $67 \%$ (71) raramente. Esse dado coincide com o encontrado na literatura (Gilbert et al., 2012; Gilbert, 2013, 2019), o que permite levantar a hipótese de uma tendência de relacionamentos de pessoas com maior compaixão pelos outros perceberem menor fluxo de compaixão vinda dos outros. Segundo os autores, essas pessoas podem ser muito doadoras e, devido ao pouco espaço para os outros também os serem, percebem que não têm reciprocidade nos relacionamentos. Durante as sessões e as respostas das questões qualitativas 5 e 6 , os participantes fizeram comentários convergentes às hipóteses dos autores, embora não tenha sido realizada medida de engajamento no presente estudo (Gilbert et al., 2012; Gilbert, 2013, 2019).

A respeito dos grupos terem sido compostos majoritariamente por profissionais da saúde, Gre- enberg (2020) lembra que a falta de suporte social pós-trauma e a exposição a fatores estressores durante a recuperação do trauma são fatores de risco fortemente preditivos de consequências psicológicas negativas. Pode-se supor que a duração indefinida da pandemia e os fatores agravantes relacionados com a falta de estrutura para o atendimento da população mostrou-se traumatizante para esses profissionais. Os relatos dos participantes apontaram a carência de um espaço como essa intervenção e expressaram forte gratidão pela oportunidade de participar da intervenção, o que pode ter influenciado os resultados.

Outro dado que deve ser mencionado é a prevalência de mulheres entre os participantes da intervenção. Neff (2021) afirma que a compaixão é vista como uma habilidade ou atributo reservado às mulheres, visto que é muito associada à mansidão e ternura. Para as mulheres é autorizado que sejam fraternas, pois isso é socialmente esperado e inclusive incentivado. Já os homens, devido a esse estigma social dos papeis de gênero, parecem procurar menos esse tipo de intervenção, além de entrarem nos grupos mais receosos de demonstrarem tais sentimentos. Sustentada por seus estudos sobre a autocompaixão feroz, ou seja, a habilidade de se expressar com assertividade e força, Neff (2021) defende que é necessário mudar essa visão. Na mesma direção, Gilbert (2019) aponta as grandes qualidades da compaixão como sabedoria, força/coragem e comprometimento. Ele afirma que a compaixão não pode ser confundida com ser "bonzinho" ou doce, mas muitas vezes ser firme e forte (Gilbert, 2019). Ao longo da intervenção, observou-se que a manifestação verbal dos participantes do sexo masculino foi menor, mesmo que isso não se refletisse nas práticas propostas para a semana e nos beneficios percebidos da intervenção. Hipotetiza-se, baseado na literatura apresentada e no relato de percepção dos participantes, uma possivel influência dessa imagem distorcida da compaixão reservada ao público feminino.

Os resultados quantitativos indicam relevantes mudanças nos escores dos instrumentos, corroborados pelos dados obtidos através das 
análises qualitativas. De toda forma, por se tratar de um método ecologicamente válido, as conclusões possiveis para este estudo são de que uma intervenção online baseada em TCF tem grande potencial de efetividade, sem, no entanto, ser possivel confirmar essa hipótese a partir dos resultados alcançados.

\section{Considerações finais e perspectivas futuras}

Esse estudo se propôs a relatar a experiência de uma intervenção online de Terapia Focada na Compaixão em Grupo, verificando se ela poderia impactar na percepção dos niveis de depressão, ansiedade, estresse e autocompaixão. Além disso, objetivou oferecer uma perspectiva de atuação online, ainda lacunar para esse tipo de programa. As medidas de resultado mostraram mudanças nos indices de depressão, ansiedade, estresse e autocompaixão, em alguns casos melhorando quatro vezes mais, segundo a percepção dos participantes do programa. Com base nestes dados, por se tratar de um relato de experiência, apenas é possivel afirmar que mudanças foram encontradas, porém, ensaios clínicos randomizados e controlados deverão ser conduzidos para verificar a eficácia da intervenção ofertada. Os resultados aqui encontrados permitem traçar hipóteses acerca da contribuição positiva da TFC em grupos online para o momento de pandemia e da importância da continuidade de investigação desses fatores, porém não são generalizáveis e devem ser tomados com parcimônia.

Os dados qualitativos mostraram a autoconscientização dos participantes acerca da necessidade do autocuidado e da autocompaixão nos seus três pilares (mindfulness, senso de humanidade e bondade consigo), bem como das qualidades da compaixão (sabedoria, força/coragem e comprometimento). Tais percepções auxiliam a traçar hipóteses exploratórias para propostas de estudos futuros, bem como podem sensibilizar os profissionais para avaliações e para alertarem-se a tais variáveis na realidade dos atendimentos psicológicos.

Em relação às limitações do estudo, é relevante ressaltar que não se trata de um ensaio clínico randomizado ou controlado e sim de um relato de experiência clínica de uma intervenção em grupo realizada para auxiliar pessoas em sofrimento durante a pandemia da COVID-19 no nosso País. Alguns desafios, como qualidade da internet ou imprevistos no contrato com os participantes, dificultaram a participação e a coesão do grupo e podem ser considerados em estudos futuros. Entre as sugestões adicionais para pesquisas futuras, estão o estudo da viabilidade da adaptação do programa de 12 semanas para três semanas, a realização do mesmo em formato online, o uso de medidas de engajamento e estudos com amostras mais amplas e diversas.

\section{Referências}

Banerjee, D., \& Rai, M. (2020) Social isolation in Covid-19: The impact of loneliness. International Journal of Social Psychiatry, 66(6), 525-527. https://doi. org/10.1177/0020764020922269

Bardin, L. (1996). Análise de conteúdo (Trad. Rego L.A., Pinheiro A.). Edições 70. (Obra original publicada em 1977).

Conselho Federal de Psicologia. Resolução CFP $n$. 11/2012 (Revogada pela Resolução CFP n. 11/2018). 2012. Recuperado em 25 jun. 2020, em https://site. cfp.org.br/wp-content/uploads/2012/07/ResoluXo_CFP_nx_011-12.pdf

Conselho Federal de Psicologia. Resolução CFP $n$ 04/2020. 2020. Recuperado em 25 jun. 2020, em https://leisinstitucionais.s3.amazonaws.com/originais/ conselho_federal_de_psicologia-br/2020/resexepro-4-2020-cfp-br.pdf

Demarzo, M., Montero-Marin, J., Puebla-Guedea, M., Navarro-Gil, M., Herrera-Mercadal, P., Moreno-González, S., Calvo-Carrión, S., Bafaluy-Franch, L., \& Garcia-Campayo, J. (2017). Efficacy of 8- and 4-Session Mindfulness-Based Interventions in a Non-clinical Population: A Controlled Study. Frontiers in Psychology, 8(8), 13-43. https://doi. org/10.3389/fpsyg.2017.01343

Depue R. A., Morrone-Strupinsky, J.V. (2005) A neurobehavioral model of affiliative bonding: implications for conceptualizing a human trait of affiliation. Behavioral and Brain Sciences, 28(3), 313-349. https://doi. org/10.1017/S0140525X05000063

Du Toit, A. (2020). Outbreak of a novel coronavirus. Nature Reviews Microbiology, 18, 123. https://doi.org/10.1038/ S41579-020-0332-0

Ferguson, C. J. (2009). An effect size primer: A guide for clinicians and researchers. Professional Psychology: Research and Practice, 40(5), 532-538. http://doi. org/10.1037/a0015808 
Gilbert, P. R., Choden, M. (2014). Mindful compassion: How the science of compassion can help you understand your emotions, live in the present, and connect deeply with others. New Harbinger Publications.

Gilbert, P. R. (2009). Introducing compassion-focused therapy. Advances in Psychiatric Treatment 15(3), 199-208. https://doi.org/10.1192/apt.bp.107.005264

Gilbert P, R. (2010). The compassionate mind: A new approach to life's challenges. Constable.

Gilbert, P. R. (2014) The origins and nature of compassion focused therapy. British Journal of Clinical Psychology, 53(1), 6-41. https://doi.org/10.1111/bjc.12043

Gilbert, P. R. (2015). The evolution and social dynamics of compassion. Social and Personality Psychology Compass, 9(6), 239-254. https://doi.org/10.1111/spc3.12176

Gilbert, P. R., Catarino, F. B., Duarte, C., \& Matos, M. (2017). The development of compassionate engagement and action scales for self and others. Journal of Compassionate Health Care, 4(4). https://doi.org/10.1186/ s40639-017-0033-3

Gilbert, P. R., \& Procter, S. (2006). Compassionate mind training for people with high shame and self-criticism: A pilot study of a group therapy approach. Clinical Psychology and Psychotherapy, 13(6), 353-379. https:// doi.org/10.1002/cpp.507

Gilbert, P. R., McEwan, K., Gibbons, L., Chotai, S., Duarte, J., \& Matos, M. (2012) Fears of compassion and happiness in relation to alexithymia, mindfulness, and self-criticism. Psychology and Psychotherapy, 85(4), 374390. https://doi.org/10.1111/i.2044-8341.2011.02046.x

Gilbert, P.R. (2019). Terapia Focada na Compaixão Coleção Avanços - Terapias de Terceira Onda. Hogrefe.

Greenberg, N. (2020) Mental health of health-care workers in the COVID-19 era. Nature Reviews Nephrology, 16, 425-426. https://doi.org/10.1038/s41581-020-0314-5

Huang, C., Wang, Y., \& Li, X. (2020). Clinical features of patients infected with 2019 novel coronavirus in Wuhan, China. Lancet, 395, 497-506. https://doi.org/10.1016/ S0140-6736(20)30183-5

Kelly, A. C., Zuroff, D.C., Foa, C. L., \& Gilbert, P. R. (2010). Who benefits from training in self-compassionate self-regulation? A study of smoking reduction. Journal of Social and Clinical Psychology, 29(7), 727-755. https:// doi.org/10.1521/jscp.2010.29.7.727

Kirby, J., Petrochhi, N., \& Gilbert, P. R. (2018). Programa de 12 semanas de Terapia Focada na Compaixão. Não publicado e enviado pelos autores.

Krieger, T., Reber, F., von Glutz, B., Urech, A., Moser, C. T., Schulz, A., \& Berger, T. (2018). An Internet-Based Compassion-Focused Intervention for Increased Self-Criticism: A Randomized Controlled Trial. Behavior Therapy, 50(2), 430-445. https://doi.org/10.1016/i.beth.2018.08.003

Lovibond, P. F, Lovibond, S. H. (1995). The structure of negative emotional states: comparison of the Depression Anxiety Stress Scales (DASS) with the Beck Depression and Anxiety Inventories. Behaviour Research and Therapy, 33(3), 335-343. https://doi.org/10.1016/0005-7967(94)00075-u
Lu, W., Wang, H., Lin, Y., \& Li, L. (2020). Psychological status of medical workforce during the COVID-19 pandemic A cross-sectional study. Psychiatry Research, 288 (11), 29-36. https://doi.org/10.1016/j.psychres.2020.112936

Neufeld, C. B., Rebessi, I. P., Fidelis, P. C. B., Rios, B. F., Scotton, I. L., Bosaipo, N. B., Mendes, A. I. F., Szupszynski, K. P. D. R. (2021, no prelo). LaPICC contra covid-19: relato de uma experiência de terapia cognitivo-comportamental em grupo online. Psico.

Neff, K. D. (2003). The development and validation of a scale to measure self-compassion. Self and Identity, 2(3), 223-250. https://doi.org/10.1080/15298860309027

Neff, K. (2021). Fierce Self-Compassion: how women can harness kindness to speak up, claim their power and thrive. Penguin Random House.

Shapira, L. B., Mongrain, M. (2010). The benefits of self-compassion and optimism exercises for individuals vulnerable to depression. Journal of Positive Psychology, 5(5), 377-389. https://doi.org/10.1080/17439760.2010.516763

Souza, L. K., Hutz, C.S. (2016). Adaptation of the self-compassion scale for use in Brazil: evidences of construct validity. Temas em Psicologia, 24(1), 159-172. https://doi.org/10.9788/TP2016.1-11

Vignola, R. C. Tucci, A. M. (2014) Adaptation and validation of the depression, anxiety and stress scale (DASS) to Brazilian Portuguese. Journal of Affective Disorders, 155. 104-109. https://doi.org/10.1016/i.jad.2013.10.031

Zu, Z. Y., Jiang, M. D., Xu, P. P., Chen, W., \& Ni, Q. Q. (2020). Coronavirus disease 2019 (COVID-19): a perspective from China. Radiology, 296, 15-25. https://doi. org/10.1148/radiol.2020200490

\section{Nazaré de Oliveira Almeida}

Doutoranda do Programa de Pós-graduação em Psicologia da Faculdade de Filosofia, Ciências e Letras de Ribeirão Preto, Universidade de São Paulo (USP), em Ribeirão Preto, SP, Brasil; vinculada ao Laboratório de Pesquisa e Intervenção Cognitivo-Comportamental, Departamento de Psicologia.

\section{Isabela Pizzarro Rebessi}

Mestranda do Programa de Pós-graduação em Psicologia da Faculdade de Filosofia, Ciências e Letras de Ribeirão Preto, Universidade de São Paulo (USP), em Ribeirão Preto, SP, Brasil; vinculada ao Laboratório de Pesquisa e Intervenção Cognitivo-Comportamental. Departamento de Psicologia.

\section{Karen Priscila Del Rio Szupszynski}

Pós-doutorado em Psicobiologia pelo Departamento de Psicobiologia da Universidade Federal de São Paulo (USP); professora da Universidade Federal da Grande Dourados (UFGD), em Dourados, MS, Brasil e da Pontifícia Universidade Católica do Rio Grande do Sul, em Porto Alegre, RS, Brasil. 


\section{Carmem Beatriz Neufeld}

Pós-doutorado em Psicologia pela Universidade Federal do Rio de Janeiro (UFRJ). Livre-docente em Terapia Cognitivo-comportamental pelo Departamento de Psicologia da Faculdade de Filosofia, Ciências e Letras de Ribeirão Preto da Universidade de São Paulo (USP), em Ribeirão Preto, SP, Brasil.

\section{Endereço para correspondência}

Nazaré de Oliveira Almeida

Rua da Cosmoética, 1635, Chalé 11

Cognópolis, 85856-852

Foz do Iguaçu, PR, Brasil

Os textos deste artigo foram revisados pela Poá Comunicação e submetidos para validação do(s) autor(es) antes da publicação. 